\title{
Impact de la migration sur la croissance économique dans les pays de l'OCDE
}

Impact of Immigration on Economic Growth in the OECD Countries

Impacto de la inmigración sobre el crecimiento económico en los países de la $O C D E$

\section{Leila Ben Ltaief}

\section{OpenEdition}

Journals

\section{Édition électronique}

URL : https://journals.openedition.org/remi/11922

DOI : $10.4000 /$ remi. 11922

ISSN : $1777-5418$

\section{Éditeur}

Université de Poitiers

\section{Édition imprimée}

Date de publication : 1 décembre 2018

Pagination : 167-193

ISBN : 979-10-90426-63-4

ISSN : 0765-0752

Référence électronique

Leila Ben Ltaief, «Impact de la migration sur la croissance économique dans les pays de l'OCDE », Revue européenne des migrations internationales [En ligne], vol. 34 - n4 | 2018, mis en ligne le 01 janvier 2021, consulté le 14 avril 2022. URL : http://journals.openedition.org/remi/11922 ; DOI : https:// doi.org/10.4000/remi. 11922 


\title{
Impact de la migration sur la croissance économique dans les pays de l'OCDE
}

\section{Leila Ben Ltaief ${ }^{1}$}

\begin{abstract}
À l'heure où la xénophobie gagne du terrain dans les pays développés, les migrations internationales deviennent un sujet de débat quotidien. Les migrations en provenance des pays extérieurs à l'Organisation de coopération et de développement économiques (OCDE) ont progressé. Les données de 2015 montrent que les flux migratoires permanents ont nettement augmenté pour la deuxième année consécutive. D'après I'Insee (Institut national de la statistique et des études économiques), est considérée comme migrante la personne née à l'étranger, résidente dans un pays d'accueil, de nationalité étrangère ou ayant pris la nationalité du pays d'accueil. 4,8 millions de personnes ont immigré vers ces pays, légèrement plus qu'au cours du pic de 2006 et $10 \%$ de plus qu'en 2014 (OCDE, 2016). Cette réalité s'est traduite par une opposition aiguë dans I'opinion publique qui s'est intensifiée en Europe. Plusieurs pays ont tendance à réduire la migration légale et à lutter fortement contre la clandestinité, car la crise économique et financière de 2008 a beaucoup pesé sur leurs finances publiques (OCDE, 2013).
\end{abstract}

Paradoxalement, les économistes considèrent que cette vague d'immigrés ne présente aucun danger pour les pays d'accueil, même en période de faible croissance et de chômage élevé. Au contraire, elle constitue une aubaine dont ils doivent profiter (Kharlamova et Sitnitskiy, $2016: 210-234$ ). Leur réception est certes coûteuse pour les États, à court terme, car ils doivent investir dans des structures d'accueil et des politiques d'accompagnement social et sanitaire, mais les bénéfices seront cueillis à moyen terme. Tout l'enjeu est de réduire le temps nécessaire pour que les immigrés s'intègrent plus vite au marché du travail.

Ce phénomène migratoire incontrôlé est, en fait, le résultat d'une inégalité de richesse entre les pays du Sud et ceux du Nord. L'excès de population et de pauvreté se concentre pleinement dans les 175 pays en développement (pays du Sud) qui sont loin d'avoir les infrastructures nécessaires au développement harmonieux de leurs économies. Quant aux vingt-cinq pays développés (pays

1 Maître assistante et chercheuse en sciences économiques, Université de Sousse, Faculté des Sciences Économiques et de gestion, Cité Riadh, 4023 Sousse, Tunisie ; benltaiefleila@yahoo.fr 
du Nord), ils accumulent et profitent de la plupart de la richesse mondiale pour une population vieillissante.

Les mutations économiques et institutionnelles des pays d'accueil influencent largement le degré d'activité de la population migrante (nationalité, qualification, taux de participation, répartition sectorielle et taux de chômage). En 2015, près de 2 millions de personnes ont acquis la nationalité d'un pays de I'OCDE, soit $2,5 \%$ de la population étrangère en moyenne ; I'acquisition de la nationalité ou le changement des conditions d'accès des allochtones au marché du travail modifient la masse active étrangère. De même, l'accès libre et la résidence aisée des travailleurs entre quelques pays membres de I'OCDE expliquent la hausse des flux migratoires enregistrés (accords entre I'Irlande et le Royaume-Uni, I'Union européenne, le MERCOSUR). Faciliter l'intégration de nombreux migrants sur le marché du travail et l'éducation et le devenir professionnel de leurs enfants sont devenus, certes, une priorité majeure des pouvoirs publics.

Le déséquilibre démographique entre les pays avancés et ceux en développement constitue un des facteurs du dynamisme migratoire actuel et à venir et nécessite de réétudier l'ordre économique mondial. Dans quelques sociétés européennes telles que I'Allemagne, la Suisse, I'Italie et certains pays scandinaves, la migration contribue à maintenir le niveau de la population active et pour certaines, elle constitue le socle de toute croissance démographique (Steiner et al., 2013 et Tableaux 1a et b).

Avec une population vieillissante et un taux de fécondité faible, on estime que jusqu'en 2050, près de 56 millions de travailleurs immigrés seront nécessaires en Europe pour répondre aux besoins de leurs économies en maind'œuvre (Commission européenne, 2016). La migration constitue, donc, une composante essentielle du futur équilibre démographique de certaines nations, qui peut alimenter la croissance et la compétitivité de certains secteurs. C'est à une migration jeune, instruite, féminisée, qualifiée que les pays de I'OCDE font actuellement appel. La part de leurs travailleurs compétents représente aujourd'hui près de $40 \%$ des flux migratoires dont les deux tiers d'entre eux sont originaires des pays en développement (OCDE, 2016). L'éducation est une institution qui favorise la participation des migrants à la vie sociale. Elle contribue à améliorer leur niveau de qualification qui peut être considérée comme un accroissement de la qualité du facteur travail. En fait, la composition éducative de la population migrante est influencée par les politiques de migration sélectives. Le Royaume-Uni, le Danemark et les Pays-Bas ont récemment réformé leur système de migration pour donner plus de priorité aux migrants instruits. La plupart des pays européens ont également mis en œuvre des programmes de migration spécifiques pour attirer des travailleurs étrangers hautement qualifiés (Chaloff et Lemaitre, 2009). Cette tendance est susceptible de se poursuivre et de se renforcer dans l'avenir. 
Tableau 1(a) : Évolution de l'accroissement naturel et de la migration nette

\begin{tabular}{lccccc}
\hline Pays & $1990-1995$ & $1995-2000$ & $2000-2005$ & $2005-2010$ & $2010-2015$ \\
\hline Canada & & & & \\
M.N. & 699 & 762 & 1027 & 1230 & 1176 \\
A.N. & 938274 & 640814 & 527728 & 639935 & 637891 \\
\hline Allemagne & & & & \\
M.N. & 3233 & 744 & 1 & 32 & 1250 \\
A.N. & -577991 & -461263 & -650341 & -843134 & -99676 \\
\hline Italie & 153 & & & & \\
M.N. & -40266 & -197029 & -114026 & -75656 & -318591 \\
A.N. & & 224 & 1624 & 1006 \\
\hline
\end{tabular}

Légende : M.N. : migration nette ; A.N. : accroissement naturel.

Source : OCDE, 2018.

Tableau 1(b) : Évolution de l'accroissement naturel et de la migration nette

\begin{tabular}{|c|c|c|c|c|c|}
\hline Pays & 1990-1995 & $1995-2000$ & $2000-2005$ & $2005-2010$ & $2010-2015$ \\
\hline \multicolumn{6}{|l|}{ Suisse } \\
\hline M.N. & 247 & 65 & 186 & 345 & 382 \\
\hline A.N. & 95716 & 83321 & 56902 & 77054 & 85862 \\
\hline \multicolumn{6}{|l|}{ Belgique } \\
\hline M.N. & 115 & 67 & 248 & 267 & 270 \\
\hline A.N. & 68282 & 39289 & 45496 & 101338 & 99216 \\
\hline \multicolumn{6}{|l|}{ Suède } \\
\hline M. nette & 156 & 58 & 142 & 262 & 273 \\
\hline A. naturel & 111153 & -12906 & 16156 & 89681 & 124503 \\
\hline \multicolumn{6}{|l|}{ Norvège } \\
\hline M.N. & 47 & 59 & 67 & 174 & 236 \\
\hline A.N. & 7251 & 73011 & 65416 & 92862 & 84051 \\
\hline \multicolumn{6}{|l|}{ Danemark } \\
\hline M.N. & 65 & 75 & 46 & 88 & 97 \\
\hline A.N. & 26784 & 31133 & 33103 & 45278 & 21283 \\
\hline \multicolumn{6}{|l|}{ Finlande } \\
\hline M.N. & 47 & 22 & 32 & 71 & 107 \\
\hline A.N. & 74248 & 46152 & 37951 & 50182 & 28355 \\
\hline
\end{tabular}

Légende : M.N. : migration nette ; A.N. : accroissement naturel.

Source : OCDE, 2018. 
L'arrivée d'une population sur un territoire apporte une source potentielle de travail supplémentaire qui permet, théoriquement, au pays d'accueil d'accroître sa croissance (Chojnicki et Ragot, 2012). Cette perception n'est justifiée que si les immigrés sont sélectionnés sur la base de leur niveau d'éducation, de qualification et d'expériences professionnelles. La grille de choix porte essentiellement sur le niveau de scolarité, d'âge et sexe, d'ancienneté et des connaissances linguistiques (Akbari et Mac Donald, 2014 : 577-917 ; Picott et al., 2014). Ces changements dans les tendances et les politiques migratoires nous incitent alors de repenser à l'impact économique de la migration sur la croissance économique. Ces dernières années, des analyses empiriques ont été florissantes dans deux domaines clés susceptibles d'influer l'opinion publique sur les migrations. II s'agit de l'impact du marché du travail des migrants (Borjas, 2003 : 1335-1374 et 2009 ; Angrist et Kugler, 2003 : 302-331; Lubotsky, 2007 : 820-867 ; Ottaviano et Peri, 2008) et de I'incidence budgétaire de la migration (Storesletten, 2000 : 300-323), (Storesletten, 2003 : 487-506 ; Hansen et Lofstrom, 2003 : 74-98). Cependant, le débat est relativement muet concernant une troisième zone d'intérêt assez importante qui est l'effet des migrations sur la croissance, question posée dans ce travail. Certaines études ont abordé des thèmes proches, mais elles ont rarement réuni la problématique de l'éducation et celle de la croissance. Ortega et Peri (2009) établissent un modèle de gravité pour quatorze pays de I'OCDE pendant la période 1980-2005. Ils découvrent que la migration accroît le PIB mais n'a pas d'effet sur le PIB par habitant. D'Albis et al. (2013) proposent une évaluation quantitative des interactions entre le PIB par habitant et le taux de chômage, et la migration permanente en France métropolitaine durant la période 1994-2008. Les résultats empiriques montrent que le taux de migration, et en particulier celui de la migration familiale, a un effet positif et significatif sur le PIB par habitant, tandis que les effets de la migration sur le chômage sont marginaux.

Cet article propose un diagnostic précis des migrations dans un contexte de mondialisation. Il analyse leurs effets sur le marché du travail, le chômage et la croissance économique de pays de I'OCDE. II permet de répondre aux deux questions suivantes : doit-on considérer les nouveaux arrivants étrangers comme un stimulant de la croissance économique ? Et, est-il important de les choisir selon leur niveau d'instruction et de compétence ? La première section analyse les dynamiques migratoires et les politiques de main-d'œuvre dans cette zone. La seconde section examine l'importance des migrations sur le chômage ainsi que la mauvaise compréhension de l'ajustement macroéconomique. Quant à la section trois, elle décrit les résultats empiriques de l'impact des différentes variables de migration sur le PIB per capita et détermine la proportion de migrants qui constitue le vrai moteur de développement. La section quatre conclut par un résumé des constats.

\section{Dynamisme de la migration internationale et politique de main-d'œuvre}

D'après les données de l'OCDE (2016), la part des immigrés dans la population totale de l'OCDE s'est accrue, passant de 8,49\% en 2005 à 10,04 \% en 2015. En 2015, un grand nombre d'allochtones en Europe se trouvent en Allemagne (7,5 millions), au Royaume-Uni (5,4 millions), en Italie (5 millions), en Espagne 
(4,5 millions) et en France (4,4 millions). Il représente $76 \%$ du nombre total de non-nationaux résidants dans I'Union européenne. Aujourd'hui, I'Allemagne est le deuxième pays au monde, derrière les États-Unis, à attirer le plus d'immigrés. Les décideurs politiques cherchent alors à être plus sélectifs dans leur choix en s'assurant que les nouveaux arrivants soient plus instruits et compétents. Mais pour que ce choix débouche sur une solution viable et pérenne, il faut que les immigrés soient bien intégrés dans l'économie des pays d'accueil.

Quels que soient le motif de leur entrée et l'ancienneté de leur présence dans la plupart des pays membres de l'OCDE, les immigrés occupent une place significative dans la population totale et sur le marché du travail. En 2014, les allochtones représentent $20,53 \%$ de la population active au Royaume-Uni et plus de $20 \%$ aux États-Unis et en Allemagne (OCDE et Eurostat, 2015 et Graphique 1).

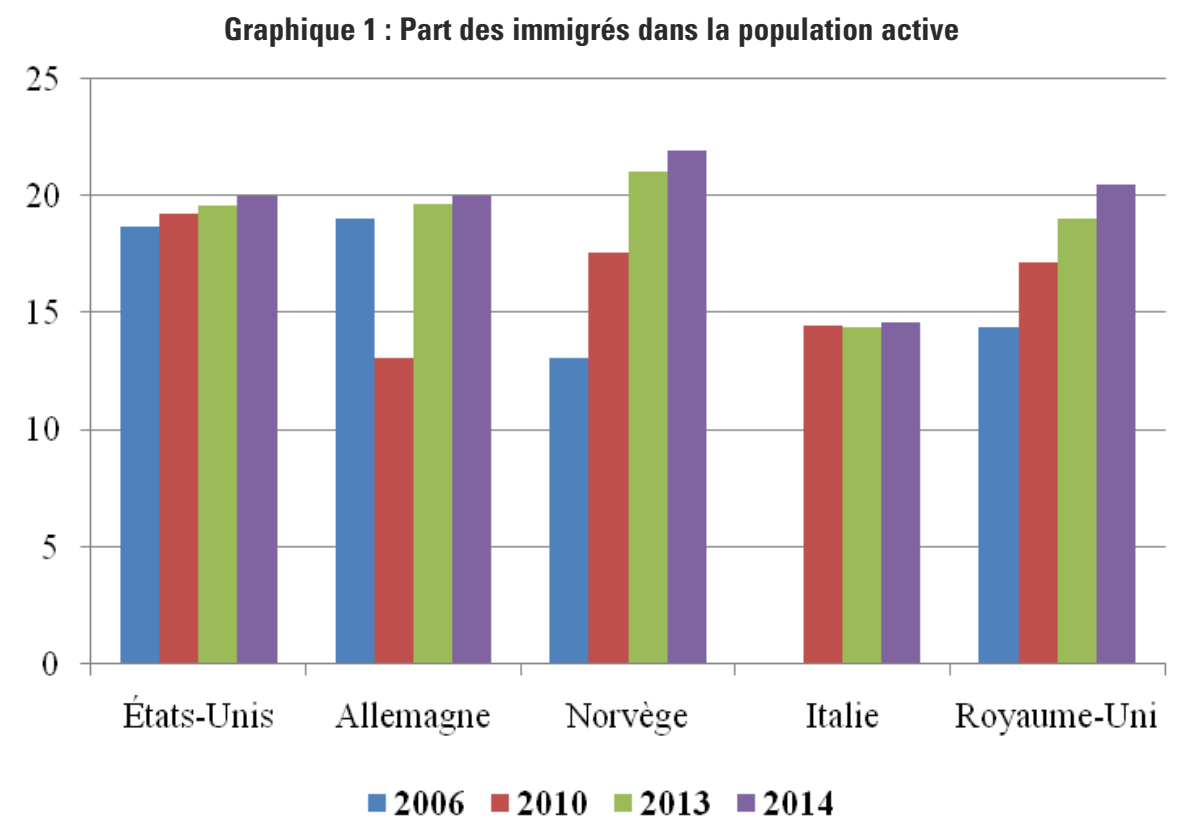

Source : OCDE, 2015.

En 2015, on compte environ 244 millions migrants internationaux dans le monde (dont 30 millions sont en situation irrégulière), contre 720 millions à l'intérieur des pays. Ils représentent 3,3\% de la population mondiale (Mouhoud, 2017). Le réchauffement de la planète et les désastres environnementaux ; et la multiplication des conflits et des guerres au Moyen-Orient et en Afrique ont intensifié les flux migratoires internationaux. Durant cette année, la migration s'établit à 4,8 millions de personnes dans les pays de l'OCDE. L'augmentation est essentiellement due aux migrations intra-européennes en libre circulation qui correspondent à l'augmentation du nombre de réfugiés humanitaires ou à des flux migratoires d'installation; les migrations saisonnières, transfrontalières et les travailleurs détachés sont donc exclus. 
Dans la plupart des pays de I'OCDE, la migration provient, via la mer Méditerranée et les Balkans, de I'Afrique, le Moyen-Orient et l'Asie du Sud. Les flux d'Asie sont croissants où un nouveau migrant sur dix provient de la Chine et $4 \%$ de I'Inde. La Roumanie et la Pologne occupent désormais la seconde position dans cette zone avec plus de $5 \%$ des flux pour chaque pays (OCDE, perspectives des migrations internationales, 2017). Cette présence étrangère demeure très variable selon les pays et dépend essentiellement des traditions migratoires, des réseaux établis par les communautés déjà sur place, des possibilités d'emploi et de la proximité géographique du pays d'origine. Aux États-Unis, c'est la présence des Mexicains qui prédomine où ils se classent au premier rang (Davis, 1974 ; Monnet, 2006). Quant à I'Union européenne, sa part d'immigrés originaires des pays tiers a augmenté et certaines nationalités ont apparu ou gagné en importance et en nombre, comparativement à d'autres, présentes depuis plus longtemps. Le Luxembourg, I'Irlande, la Belgique et le Portugal enregistrent une forte proportion de ressortissants communautaires dans les nombres globaux d'étrangers. Dans la population comme dans l'emploi, les Algériens, les Marocains et les Sénégalais restent les premières nationalités d'origine des allochtones en France (Pan Ké Shon et Verdugo, 2015 : 823-840) alors qu'aux Pays-Bas, ils sont principalement les originaires de l'ex-Yougoslavie. Dans les pays nordiques, la part de ressortissants des pays voisins a diminué en Finlande, en Norvège et en Suède, tandis que de nouvelles communautés étrangères ont vu leurs nombres s'élever (Giang et Kazuko, 2015). II s'agit des Asiatiques (Pakistanais, Vietnamiens, Iraniens et Sri Lankais) et des Turcs en Norvège et en Suède, et des ressortissants de I'ex-Yougoslavie en Norvège, en Suède et en Finlande (Friberg et Eldring, 2013). En 2015, le taux d'emploi des immigrés de pays de l'OCDE était de 70,8 \% contre $73 \%$ des natifs (OCDE, 2015). Cette proportion s'accentue tendanciellement, en dépit du choc conjoncturel de 2008. Ces taux étaient respectivement égaux à $62,1 \%$ et $65,2 \%$ dans I'Union européenne (Cf. Graphique 2).

Au cours de cette année, I'Islande $(80,7 \%)$, la Suisse $(76,5 \%)$ et la NouvelleZélande $(73,5 \%)$ ont dégagé les taux d'emploi des étrangers les plus élevés dans I'OCDE et ceci suite à la mise en application de différentes politiques d'intégration. Les pays proches du plein emploi - comme l'Allemagne, le Royaume-Uni, la Suisse, la Norvège, les États-Unis et I'Islande - ont un intérêt économique à accueillir des immigrés, particulièrement des réfugiés, afin de minimiser les difficultés de recrutement qui apparaissent dans certains secteurs d'activité (Hinger, 2017 ; Jakob, 2016). Cette migration réajuste, par conséquent, les offres et les demandes d'emploi et crée un chômage positif et souhaité de la part des employeurs. L'objectif de ces derniers, qui craignent pour leur compétitivité et leurs profits, est d'éviter des augmentations de salaire dans une situation très voisine du plein emploi. Les taux d'emploi des migrants venus de pays ne faisant pas partie de I'OCDE sont inférieurs à ceux des citoyens des pays de I'OCDE (Cf. Graphique 2). 
Graphique 2 : Taux d'emploi des immigrés et des natifs (EU-28 et OCDE) (en \%)

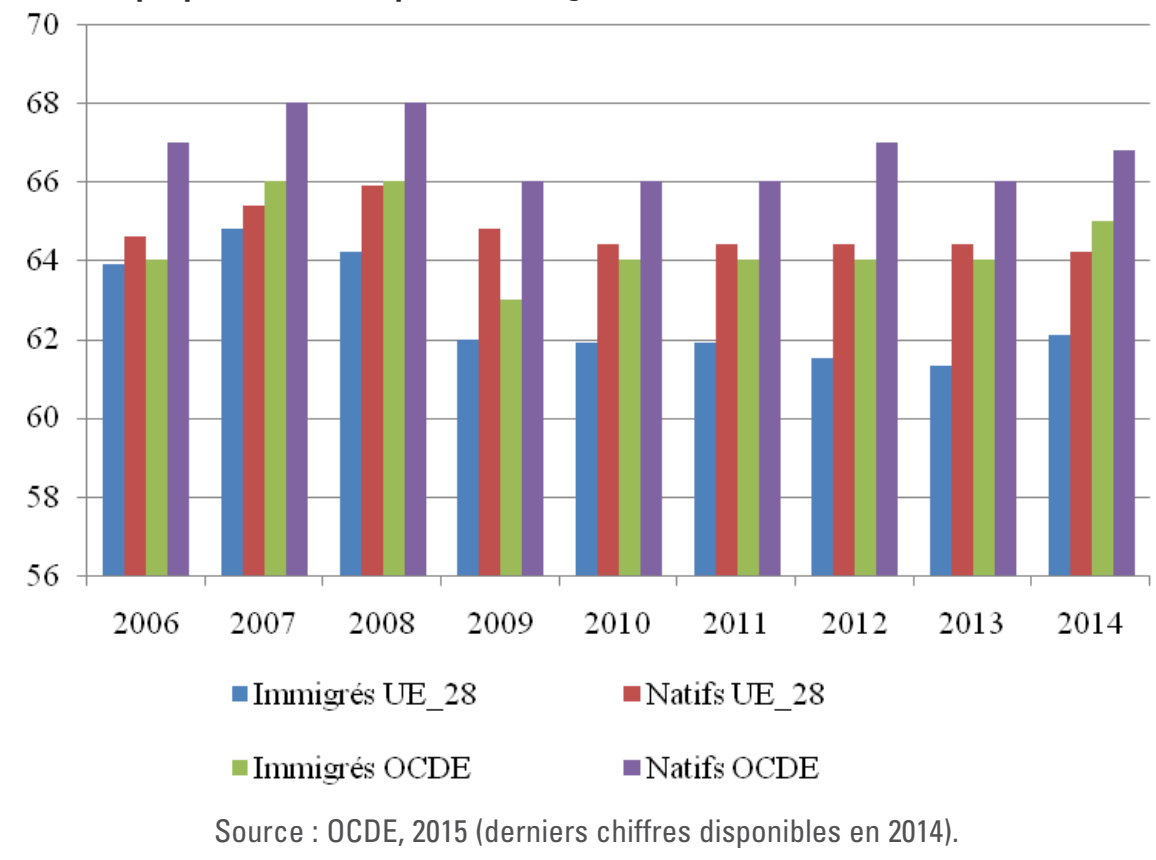

Les ressortissants de pays tiers diplômés du supérieur ont de plus grandes difficultés à trouver un emploi que leurs semblables nationaux. Le tableau 2 montre que le taux d'emploi des migrants actifs ayant un niveau d'étude élevé a baissé en 2015 en Belgique, en France, en Italie et dans certains pays nordiques (Danemark, Finlande et Norvège), comparativement à l'année 2010. Bien que les actifs immigrés soient employés dans toutes les professions, ils sont largement recrutés comme ouvriers et manœuvres. Ils sont dès lors moins présents que les natifs dans les professions intermédiaires de même que dans les professions intellectuelles supérieures et chez les cadres. Cette employabilité étrangère est le plus souvent concentrée dans quelques secteurs d'activité. On constate une tendance à sa répartition dans I'ensemble de l'appareil productif, notamment dans le secteur tertiaire. Leur présence augmente également dans les secteurs d'éducation, de santé et d'action sociale, en raison de la progression constante du niveau de formation des migrants depuis plusieurs années (OCDE, 2016). Cet exercice fournit une meilleure compréhension du marché du travail des pays de I'OCDE en présence des flux massifs de migrants ainsi que les déséquilibres qui s'y produisent. Nous le voyons comme un outil pour reconnaître les signes de véritables pénuries de main-d'œuvre, mais également pour alimenter la réflexion sur les besoins et les façons d'intervenir par une migration sélective des travailleurs instruits. Dans ce qui suit, nous cherchons l'impact des flux d'immigrés sur le chômage dans les pays d'accueil et si ces derniers sont la cause principale de l'inactivité des nationaux. 
Tableau 2 : Taux d'emploi des migrants actifs par niveau d'études (en \%)

\begin{tabular}{|c|c|c|c|c|c|c|}
\hline & \multicolumn{2}{|c|}{ Niveau d'études faible } & \multicolumn{2}{|c|}{ Niveau d'études moyen } & \multicolumn{2}{|c|}{ Niveau d'études élevé } \\
\hline & 2010 & 2015 & 2010 & 2015 & 2010 & 2015 \\
\hline Autriche & 56,6 & 51,3 & 73,9 & 71,2 & 75,9 & 75,8 \\
\hline Allemagne & 55,4 & 58,8 & 71,9 & 76,4 & 76,5 & 78 \\
\hline Royaume-Uni & 45,7 & 55,9 & 72,8 & 75,2 & 80,8 & 81,2 \\
\hline Irlande & 41,9 & 47,1 & 61,7 & 64,7 & 74,1 & 74,7 \\
\hline Belgique & 41 & 41,6 & 62,1 & 61,1 & 73,5 & 73,3 \\
\hline France & 52,2 & 48 & 65,3 & 61,1 & 72,9 & 71,7 \\
\hline Italie & 62,5 & 59 & 70,3 & 66,9 & 71,7 & 69,2 \\
\hline Luxembourg & 68,7 & 64,9 & 70,3 & 69,4 & 82,7 & 83,3 \\
\hline Pays-Bas & 52,5 & 49,1 & 71 & 66,1 & 77,4 & 79,5 \\
\hline Suède & 45,9 & 52,3 & 68 & 75,2 & 75,3 & 78,3 \\
\hline Suisse & 68,9 & 69,8 & 77,1 & 79,9 & 81,3 & 83,5 \\
\hline Danemark & 54,1 & 49,1 & 67,1 & 68 & 77 & 75,4 \\
\hline Finlande & 57,5 & 52,3 & 68,7 & 64,6 & 76,5 & 68,7 \\
\hline Norvège & 58,5 & 54,4 & 73,5 & 73,5 & 85,7 & 81,4 \\
\hline Islande & 76,3 & 82,5 & 74,8 & 82,1 & 84,1 & 85,1 \\
\hline Nouvelle-Zélande & 57,9 & 62,7 & 76,7 & 77 & 80,7 & 84 \\
\hline États-Unis & 63,6 & 65,8 & 70,3 & 71,6 & 76,8 & 77,4 \\
\hline Canada & 52,2 & 56,3 & 69,3 & 69,8 & 76,6 & 77,9 \\
\hline
\end{tabular}

Source : OCDE, 2016.

\section{Migration et chômage : mauvaise compréhension de l'ajustement macroéconomique}

En 2015, 42 millions de travailleurs sont sans emploi dans les pays de I'OCDE (OCDE, 2015). Le taux de chômage dans ces pays est de 6,5\% au cours du dernier trimestre 2016, mais il reste supérieur à $20 \%$ en Grèce et en Espagne (Cf. Graphique 3).

Durant la période 2011-2015, le taux d'emploi des immigrés est resté stable ou a légèrement baissé dans cette zone. Leur taux de chômage est égal à 13,4 \% alors que celui des nationaux est de l'ordre de 8,56\%. La plus grande fragilité des migrants au chômage et leur plus faible degré d'employabilité montrent qu'ils rencontrent, surtout en Europe, des difficultés d'insertion sur le marché du travail (Monso et Gleizes, 2009). Ces différences s'expliquent par un temps d'intégration plus ou moins long surtout pour les réfugiés nouvellement arrivés, des niveaux de qualification qui ne sont pas toujours adaptés aux besoins du marché du travail du pays d'accueil, des connaissances insuffisantes de la langue du pays et des actes discriminatoires qui les empêchent d'accéder audit marché (Schuss, 2016). 
Graphique 3 : Taux de chômage des immigrés et des natifs (en \%, année 2015)

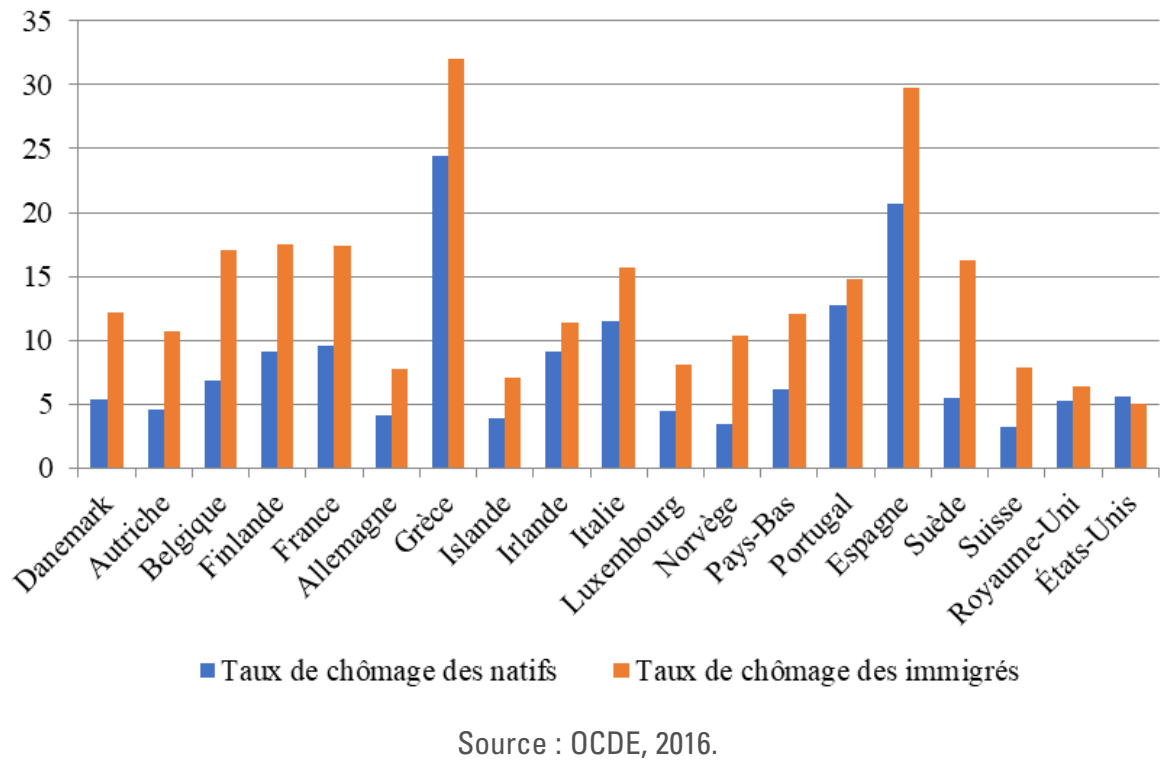

Le graphique 4 porte sur vingt-trois pays membres de I'OCDE ayant des caractéristiques structurelles très différentes, où le niveau du taux de chômage et celui de la part de migrants ne sont pas directement comparables. En 2014, les États ayant la plus forte proportion de migrants sont le Luxembourg, la Norvège, l'Autriche et la Suisse. Par contre, ceux ayant le plus fort taux de chômage sont la Grèce et I'Espagne, et dans une moindre mesure I'Irlande et I'Italie. La relation entre les variations de ces deux variables durant la période 2007-2015 montre qu'aucun pays ne se situe dans le quadrant supérieur droit du graphique, ce qui signifie qu'aucun d'entre eux ne cumule à la fois un fort taux de chômage et une forte proportion de migrants. II n'y a donc pas de lien entre migration et chômage.

Durant la période 2007-2015, la Norvège et le Luxembourg ont connu une augmentation de leur part d'immigrés de 4,4 et $4,2 \%$ respectivement. La majorité des pays étudiés ont affiché une variation située entre $-3,5$ (Estonie) et $+3,5$ (Autriche) points. En ce qui concerne les États-Unis et l'Allemagne qui sont ceux accueillant le plus d'étrangers, les variations respectives de leur part de migrants sont $-0,2$ et $+1,9 \%$. On remarque que la Norvège, le Luxembourg, les États-Unis et l'Allemagne cumulent à la fois un faible taux de chômage et une forte proportion d'étrangers. Sur l'axe du chômage, ils affichent de faibles variations égales à $+1,88$ au Luxembourg, $+1,01$ à la Norvège et $+1,55$ aux États-Unis. À une extrémité se trouve l'Allemagne où la variation du chômage est négative (-3,55 points), bien qu'elle ait accueilli le plus de migrants parmi les pays membres de la zone européenne de I'OCDE. À l'opposé la Grèce et I'Espagne enregistrent des variations très élevées de leur taux de chômage $(+18,13$ et $+16,23$ points de pourcentage), mais elles ont connu en parallèle de faibles variations de leur part de migrants dans la population totale $(+0,7$ et $-1,5)$. 


\section{Graphique 4 : Relation entre la variation du taux de chômage et la variation du ratio immigrés/population (2007-2015)}

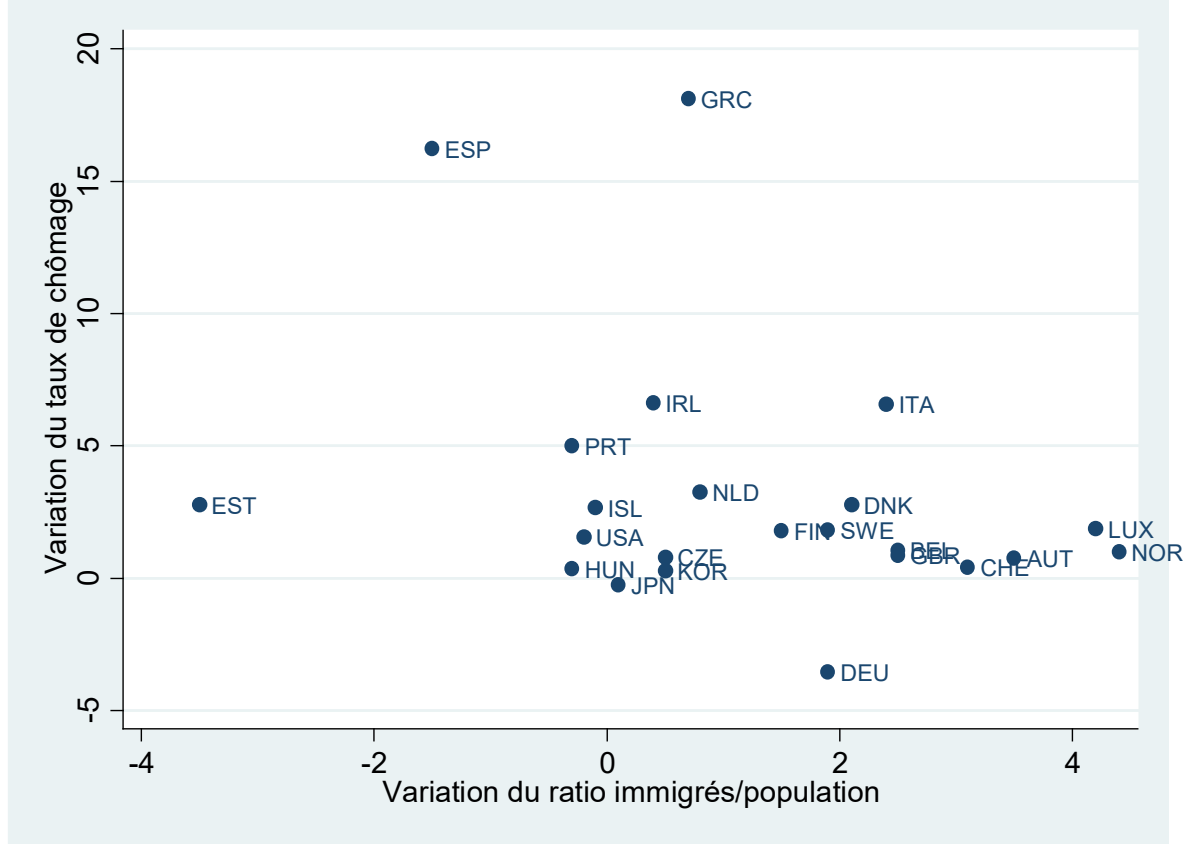

Légende : AUT : Autriche ; BEL : Belgique ; CHE : Confédération helvétique ; CZE :

République tchèque ; DEU : Allemagne ; DNK : Danemark ; ESP : Espagne ; EST : Estonie ; FIN : Finlande ; GBR : Grande-Bretagne ; GRC : Grèce ; HUN : Hongrie ; IRL : Irlande ; ISL : Islande ; ITA : Italie ; JPN : Japon ; KOR : Corée ; LUX : Luxembourg ; NLD : Pays-Bas : NOR : Norvège ; PRT : Portugal ; SWE : Suède ; USA : États-Unis.

Sources : OCDE, 2016 ; FMI, 2018.

Donc, les pays qui ont connu la hausse du chômage la plus importante (Espagne, Grèce, Irlande et Italie) ne sont pas nécessairement ceux qui ont vu la part des migrants augmenter le plus (Norvège, Luxembourg, Autriche et Suisse). En réalité, ce dernier groupe de pays qui possède un taux de chômage faible a tendance à attirer plus de migrants, tandis que les pays en crise (Espagne, Grèce, Irlande et Italie) apparaissent moins attractifs. Depuis 2007, les flux migratoires en direction des pays du sud de l'Europe ont diminué alors que l'Allemagne est devenue une destination cible. Les conséquences de la crise migratoire sur l'économie allemande et sur l'économie française sont très différentes. En effet, la population allemande décroît et le pays a tout à gagner à l'entrée de nouveaux travailleurs et consommateurs qui en outre permettront à terme de financer les pensions de retraite. Bien que la France se caractérise par un chômage important, une bureaucratie lourde et rigide et des squats insalubres, elle ne connaît pas le même déclin démographique et surtout s'oppose à investir dans le capital humain migrant. L'Allemagne apparaît désormais comme un modèle d'ouverture, de générosité et de solidarité alors que les pays de l'Europe de l'Est s'opposent fermement à l'accueil des migrants. Ainsi, le taux des réticences à 
I'accueil de migrants est de $81 \%$ en République tchèque, de $78 \%$ en Lettonie, de $77 \%$ en Slovaquie et de $70 \%$ en Lituanie (Honoré, 2015).

Les chances de trouver un emploi sont d'autant plus grandes que le taux de chômage est faible dans le pays d'accueil. L'attractivité des migrants, qui désirent travailler, est garantie par la baisse dudit taux. Les migrants et les nationaux n'occupent pas les mêmes métiers et ne sont pas parfaitement substituables. Les premiers ne sont pas en concurrence avec les seconds, ils répondent à une demande de travail différente et apaisent certaines tensions sur le marché du travail (Chojnicki et Ragot, 2012).

Le nombre d'emplois d'une économie n'est pas fixe. Il dépend principalement du fonctionnement du marché du travail (Jean et Jiménez, 2011 : 241-256). Si ce dernier est très réactif comme dans le cas allemand, il est possible de créer beaucoup d'emplois rapidement. Les nouveaux arrivants stimulent eux aussi la demande globale du pays d'accueil. Ils soutiennent l'activité des entreprises et renforcent leur demande de travail. Les citoyens étrangers sont certes des travailleurs qui vont occuper un emploi, mais ils sont simultanément des consommateurs qui, par leurs dépenses, vont engendrer la création potentielle de nouveaux emplois (Ortega et Peri, 2009). C'est donc les immigrés eux-mêmes qui vont créer les nouveaux emplois qu'ils vont occuper. Incidemment, l'inverse est aussi vrai. Quand un marché du travail fonctionne mal, il ne parvient pas à résorber le chômage, même avec peu ou pas de flux migratoires. En l'occurrence, la réalité est plus complexe et ce n'est pas la migration qui crée le chômage, mais la rigidité et le fonctionnement inadapté du marché du travail. Il convient de souligner que la migration peut avoir des effets divers sur le chômage selon l'horizon temporel étudié et selon la situation des travailleurs. De même, le degré de flexibilité du marché du travail et les contraintes législatives peuvent influer sur le lien entre la migration et le marché du travail. Ces explications mettent en cause le lien entre la migration et le chômage dans les pays de I'OCDE et s'ajoutent aux considérations théoriques qui ont été confirmées par plusieurs travaux empiriques. Citons l'étude sur l'arrivée massive de Cubains en Floride en 1980 (Card, 1990 : 245-257), le rapatriement des Algériens en 1962 (Hunt, 1992 : 556-572) et la migration des Juifs de I'Union soviétique en Israël entre 1990 et 1991 (Friedberd, 2001 : 1373-1408). Ces auteurs montrent que la migration n'a eu aucun effet sur le chômage ou que cet effet n'a été que transitoire et très faible.

\section{Importance de la migration pour la croissance : résultats économétriques et conclusions économiques}

Les résultats des estimations économétriques sont présentés dans le tableau 3 en annexe et la synthèse de la partie technique de ce travail est détaillée en annexe. II met en lumière, en plus des variables classiques, les déterminants difficilement perceptibles ou prévisibles qui impactent le PIB réel per capita. Nous réalisons quatre scénarii différents où les variables endogènes sont respectivement la part des migrants dans la population totale (P_mgr), la part de migrants à niveau d'éducation élevé (E_haut), la part de migrants à niveau d'éducation moyen (E_moyen) et la part de migrants à niveau d'éduca- 
tion faible (E_bas). Le cinquième scénario contient conjointement les variables E_haut, E_moyen et E_bas afin de démontrer la nature de la migration qui agit sur le PIB per capita.

Nous débutons notre analyse en étudiant l'impact de la production par tête sur la croissance économique l'année t par rapport l'année t-1. Nous affirmons l'absence d'une relation significative et négative entre ces deux variables pour les scénarii (1), (3) et (4). En fait, les pays de I'OCDE sont hétérogènes (propension à épargner, accès à la technologie, taux de fécondité, etc.), ce qui ne permet pas de réaliser les conditions de convergence vérifiées par Barro et Sala-I-Martin, (1995) et (Mankiw et al., 1992 : 407-437). Si le PIB réel par habitant augmente au cours du temps, les ressources matérielles des habitants tendent à augmenter, car ils ont part au succès économique par le biais des revenus du travail et du capital. Donc, pour des valeurs données des variables de choix et d'environnement (taux d'épargne, offre de travail, taux de fécondité, dépenses publiques, taux d'imposition, capacité à faire respecter la loi et les droits de propriété, degré de liberté politique, les termes de l'échange, etc.), plus le niveau de départ du PIB/habitant est faible, plus le taux de croissance attendu est élevé. Donc, tous les pays qui font un effort d'investissement sont susceptibles de connaître une croissance économique. C'est le cas de la France vis-à-vis des États-Unis entre 1950 et 1970 et du Japon entre 1960 et 1980. Cet effet correspond au principe de convergence conditionnelle. Ce résultat signifie que le niveau du $\mathrm{PIB} /$ habitant en $\mathrm{t}-1$ n'influe pas le taux de croissance économique de la période t. Ainsi, le taux de croissance de l'OCDE sera d'autant plus sensible à son niveau de départ de production/habitant $\left(\mathrm{PIB}_{\mathrm{i}, \mathrm{t}-1}\right)$ que son stock de capital humain sous toutes ses formes (niveau d'éducation, $d$ 'expérience, santé, hygiène, etc.) le sera (Lucas, 1988 : 3-42). Les efforts d'investissement en capital humain étranger (la totalité des migrants, la part des migrants à qualification moyenne ou la part des migrants à qualification faible) et en capital technique des pays, qui déterminent la convergence, n'ont pas pu combler ainsi leur retard. Ils peuvent transférer chez eux les techniques de production des pays les plus en avance, grâce à une main-d'œuvre mieux formée (migrants hautement qualifiés, scénario 2), ce qui explique la significativité du deuxième scénario. Quant au scénario (5) où les trois parts de migrants appartiennent à la même régression, le coefficient relatif au $\mathrm{PIB}_{\mathrm{i}, \mathrm{t}-1}$ est négatif et significatif $(-0,074)$. Ce résultat montre que plus le niveau de départ du PIB/habitant est faible, plus le taux de croissance attendu est élevé.

L'accroissement naturel de la population (ACC) impacte positivement la croissance économique pour les différentes régressions envisagées. Ce résultat est expliqué par des coefficients positifs et significatifs qui montrent que plus le capital humain des nationaux est important plus il est enclin à augmenter le $\mathrm{PIB} /$ habitant. Pour un dixième d'accroissement naturel supplémentaire, la valeur de la croissance augmente respectivement de 0,891,0,709, 0,913,0,9167 et 0,909 point de pourcentage, durant la période 2000-2015 (tableau 3, cinq scénarii). Ces deux phénomènes sont concomitants. Mais, la déformation des structures par âge de la population, avec le vieillissement inéluctable (Allemagne, Espagne, Italie, Japon, France, Royaume-Uni, Suède et les États-Unis), lié au déclin de la mortalité et de la fécondité dans certains pays de l'OCDE (le taux de reproduction démographique dans la plupart des pays industrialisés n'atteint pas la valeur de 2,1 nécessaire au renouvellement régulier de la population) a légitimé les tendances récentes des mouvements et des politiques migratoires actives. Les 
gouvernements encouragent la natalité et la migration qui stimulent certes les choix intertemporels de consommation et d'investissement. Si le vieillissement démographique conduit effectivement à une baisse de l'investissement et/ou un accroissement de l'épargne, il est susceptible de générer des pressions déflationnistes, déprimant davantage l'activité économique. Tel est le cas du Japon, où l'économie insulaire est tombée dans la stagnation économique précisément à l'instant même où sa population active commença à décliner, puis elle s'est enfoncée depuis dans la déflation. Avec un taux d'accroissement naturel positif et une migration bien sélectionnée, ces choix sont vraisemblablement révisés à la hausse et affecteraient positivement l'accumulation du capital physique et par la suite la croissance économique (Becker et al., 1990 : 12-37).

Parce qu'ils maintiennent leurs marchés ouverts aux échanges et à l'investissement, le Luxembourg, la Belgique, I'Irlande, les Pays-Bas ou encore la Slovénie et la République slovaque bénéficient davantage du dynamisme des échanges commerciaux. Cette conclusion a été fortement justifiée par les estimations réalisées au niveau des scénarii envisagés. Nous observons un lien fort et positif entre l'ouverture commerciale des pays étudiés et leur croissance économique. La libéralisation commerciale crée donc de la richesse. Le signe positif et significatif des coefficients de la variable en question est conforme aux intuitions théoriques et corrobore en particulier les travaux de Collier et Gunning (1999: 64-111).

Ensuite, les coefficients liés à l'accumulation du capital physique sont significatifs pour la première, la troisième, la quatrième et la cinquième spécification. Ce résultat montre que si le capital humain représente la part migrante globale (P_mgr) ou s'il symbolise les migrants à niveau d'éducation moyen (E_moyen) alors la variable investissement privé et public impacte positivement la croissance économique. La migration dans ces deux cas a des effets globalement positifs sur l'évolution des économies en matière d'investissement et en particulier sur le PIB per capita. Dans ces deux cas, si l'investissement augmente d'un dixième alors la croissance croît respectivement de 0,173 et 0,264 point de pourcentage (tableau 2, scénarii (1) et (3)). D'extensive, fondée sur l'accumulation du capital et la mobilisation du travail des nationaux et des migrants, la croissance devient intensive. À l'inverse, cet effet est fortement significatif mais négatif au moment où on tient compte des étrangers non qualifiés ou à niveau d'enseignement distinct (E_bas et scénario (5)). Ce résultat est justifié par les coefficients -2138 et -1811. À terme, cet effet négatif devrait progressivement disparaître, à mesure que le stock de capital s'ajuste à l'augmentation de cette catégorie d'offre de travail. Enfin, l'effet de la proportion de migrants bien instruits (E_haut) sur le dynamisme des investissements et par conséquent sur la croissance des pays de l'OCDE est marginal.

Quant aux investissements directs étrangers, ils sont significatifs et impactent positivement la croissance économique pour les trois premiers cas proposés, contrairement aux deux derniers qui représentent les allochtones non qualifiés ou les migrants à niveaux d'éducation différents. Ces résultats empiriques s'expliquent par le fait que dans le contexte actuel, les flux migratoires sont principalement exogènes. Ils sont déterminés par des considérations politiques dans les pays d'accueil, tandis que les flux d'IDE sont endogènes et répondent aux différentiels de rendement corrigés du risque. Comme les travailleurs ayant 
un niveau primaire sont souvent utilisés de manière intensive pour la fabrication des biens non échangeables et le travail qualifié des migrants fortement éduqués est spécifique au secteur exportateur, alors un afflux de travailleurs très ou moyennement instruits et qualifiés se traduit systématiquement par des flux entrants d'IDE. Toutefois, si les biens non échangeables nationaux et les biens importés sont relativement substituables en termes de consommation, la venue de travailleurs non qualifiés donne lieu à des sorties de capital (Ivlevs, 2006 : 50-68).

Concernant les dépenses publiques, les coefficients estimés montrent que cette variable agit positivement sur la croissance économique, en présence des migrants quel que soit leur niveau d'enseignement (sauf le troisième scénario). Ce résultat montre que les pays de I'OCDE étudiés sont majoritairement ouverts. Par conséquent, les pressions politiques conduisent à une baisse de l'effort fiscal et à un accroissement des dépenses publiques, donc à des déficits publics plus importants. L'idée est que la politique d'ouverture est le reflet d'une capacité globale à mettre en place des institutions fortes qui assurent une bonne gestion des affaires publiques. À l'inverse, leur absence conduit à des dérapages budgétaires et à une politique budgétaire au profit des groupes d'intérêt (Talvi et Vegh, 2005 : 156-190). On peut aussi envisager que le niveau des dépenses publiques soit plus réduit dans des pays ouverts sur l'extérieur et qui reçoivent des transferts de migrants. Leur effet sera par la suite positif et significatif sur le PIB par habitant.

Les résultats des estimations concernant les taux d'inflation décrivant l'environnement macroéconomique montrent que l'effet de cette variable est marginal pour l'échantillon relatif aux étrangers hautement instruits, alors qu'il est négatif et significatif pour l'échantillon relatif à la main-d'œuvre migrante moyennement qualifiée. Quant au quatrième groupe où le capital humain migrant est formé par des non éduqués, le taux d'inflation n'impacte pas la croissance économique et le signe attendu de son coefficient ne correspond pas à la théorie. Pour les scénarii (1) et (5), l'inflation agit positivement sur la croissance économique. Les coefficients sont respectivement 1,94 et 2118 . Globalement, la migration a réduit le manque de personnel instruit et qualifié dans les pays étudiés, ce qui a légèrement freiné l'évolution des salaires et par conséquent l'inflation. Une faible inflation s'est traduite à son tour par des taux de croissance réels plus élevés et un chômage toléré. Cette diminution de la pression sur les prix a été compensée par l'augmentation de la demande de consommation et de logements de la part des nouveaux migrants (Stalder, 2010).

Les estimations relatives à l'incidence de la variable institutionnelle sur le PIB per capita coïncident largement avec la littérature théorique (Eichengreen et Dincer, 2009 ; Aisen et al., 2011). Majoritairement, le " contrôle de la corruption (CC) " a un effet positif sur la croissance économique, à l'exception du scénario 5. La variable " CC " n'a pas été retenue dans la première estimation pour éviter les problèmes d'autocorrélation mentionnés précédemment. Le signe positif attendu de son coefficient est vérifié pour les quatre premiers cas. Ce résultat montre que la maîtrise de la corruption génère des effets bénéfiques pour les investissements publics et privés et diminue les coûts supportés par les entreprises, tout en augmentant la certitude quant au retour des investissements attendus. En plus, l'absence de corruption encourage les investissements directs 
étrangers, diminue les dépenses publiques et augmente les recettes fiscales. Les pays ayant un niveau de corruption faible réalisent un taux de croissance économique important, un bien-être de leurs populations et un recul de la pauvreté.

En analysant les estimations relatives aux transferts financiers des migrants générés en direction des pays d'origine en pourcentage du PIB, nous remarquons que pour les scénarii (1), (2) et (5), cette variable impacte positivement le PIB par habitant (tableau 3). Cependant, son effet s'inverse quand il s'agit du capital humain non instruit ou d'éducation moyenne. Les coefficients relatifs sont $-0,297$ et $-0,236$ (tableau 3, scénarii (3) et (4)). Nous savons que les migrants démunis d'expériences professionnelles ou moyennement qualifiés, à cause de leur niveau primaire ou secondaire faible, représentent presque la totalité de la masse migratoire dans les pays de I'OCDE. Donc, plus leur nombre est élevé plus le volume de transferts, considéré comme fuite financière vers les pays d'origine, est important. Ce phénomène est avantageux pour les pays d'origine mais défavorable à la croissance des pays d'accueil. Cette conclusion explique les signes négatifs des coefficients au niveau du troisième et quatrième scénario.

Terminons en étudiant l'effet des différentes catégories de migrants sur la croissance économique et dégageons le scénario le plus significatif de l'état actuel des pays de I'OCDE. Les variables en jeu décrivent les proportions des allochtones en fonction des niveaux d'instruction qui impactent ultérieurement leurs formations et leurs qualifications acquises. Pour les quatre premiers scénarii, les coefficients issus desdites régressions sont positifs et significatifs. Le signe attendu est donc vérifié. Ce résultat économétrique montre que, quel que soit le niveau de qualification des migrants, ces derniers interviennent à des niveaux différents pour améliorer le taux de croissance du PIB/habitant des pays de I'OCDE.

Globalement, les flux migratoires vers les pays de I'OCDE se composent simultanément des trois types de migrants. C'est pourquoi dans le scénario (5) et en plus des variables traditionnelles, nous avons introduit dans la même régression les trois composantes de migration (E_haut, E_moyen et E_bas). Ce scénario décisif nous a permis de distinguer laquelle de ces trois parts de migrants impacte positivement la croissance économique. En fait, une augmentation d'un dixième de la proportion de migrants fortement ou moyennement éduqués accroît le PIB per capita de respectivement 0,2188 et 0,2632 point de pourcentage (tableau 3, scénario (5)). La migration de ces deux formes de travailleurs influence favorablement la capacité d'innovation, l'activité entrepreneuriale et les contacts économiques internationaux, bénéfiques à l'échelle macroéconomique. D'ailleurs, dans la plupart des pays de I'OCDE, la proportion d'immigrés diplômés du supérieur dépasse celle des autochtones (Ichou, 2014 : 750-765). Par conséquent, les migrants contribuent à l'accumulation totale et par tête du capital humain, ce qui se traduit par une source potentielle de croissance endogène (OCDE, 2016). Cependant, nous en déduisons également que le poids des migrants faiblement instruits (E_bas) n'influence pas le PIB per capita. Cette variable demeure non significative là où elle présente une $p$-value égale à 0,301 (tableau 3, scénario (5), cas de E_bas). Malgré ce résultat décevant, la migration des non instruits continue en direction des pays de I'OCDE. Ce groupe d'allochtones participe, certes, à améliorer le bien-être collectif, mais le faible niveau de qualification et le fort taux de natalité le prédisposent effectivement 
à un moindre taux d'activité et à un taux de chômage plus élevé. Ils restent surreprésentés dans les emplois non qualifiés qui n'exigent pas un niveau d'instruction élevé. Si ces flux de main-d'œuvre sont bloqués, certains pays auront certainement plus de peine à répondre à la satisfaction de leurs habitants. Dans les pays de l'OCDE européens, le vieillissement de la population nécessite la mise en place de services auprès des personnes âgées (services de soins et d'assistance). Si on bloque complètement la migration de la main-d'œuvre non qualifiée ayant un niveau d'enseignement faible, on nuit au bien-être de la population et des personnes les plus démunies. Ceci est particulièrement grave dans le cas des services de santé et des systèmes de santé publique. Cette catégorie de migrants aide les personnes âgées et essaie de soulager les souffrances des pauvres. D'autre part, alors que les nationaux délaissent la formation et l'orientation vers les filières sans perspective, les immigrés jouent un rôle dans les professions en déclin et répondent aux besoins du marché de l'emploi. Ces métiers en récession recrutent des étrangers pour remplacer une partie des départs à la retraite. Cette conclusion montre que le recours à la main-d'œuvre étrangère est nécessaire à tous les échelons de qualification tant les besoins de certaines compétences sont élevés.

\section{Conclusion}

De nombreux pays de l'OCDE sont confrontés aux pénuries de main-d'œuvre dans certains secteurs. De plus, la perspective du vieillissement démographique se traduit par un manque de cotisations qui assurent l'avenir des futurs retraités. La migration est alors perçue comme un remède au moins partiel à ces deux problèmes, malgré l'existence de certaines ressources inutilisées sur le marché du travail (chômage de masse et possibilités d'élever le taux de participation des femmes). Les gouvernements des pays d'accueil recourent à cette solution où ils bénéficient $d$ 'un capital humain étranger qui arrive dans leurs pays à l'âge adulte et leur permet d'épargner le financement de scolarité ainsi que le reste des frais dépensés par le pays d'origine avant le départ des migrants. Pour contrecarrer la perception négative de I'opinion publique vis-à-vis des migrations internationales en temps de crise et bénéficier du "brain drain ", I'accueil des migrants est fondé sur des stratégies de localisation en créant des réseaux qui lient les services sociaux municipaux, les centres d'emploi et les organisations non gouvernementales et enfin des programmes sélectifs en fonction des besoins des pays d'accueil. L'ensemble de ces dispositifs a permis une participation meilleure des migrants au marché de travail et la création de nouveaux emplois. De leur côté, la majorité des pays membres de I'OCDE n'ont pas enregistré des effets indésirables à court terme qui touchent le licenciement de la main-d'œuvre nationale, la pression sur les salaires ou la hausse du chômage.

La migration est un phénomène sélectif. Les migrants sont supposés être des individus ayant des caractéristiques spécifiques. Parmi ces caractéristiques, celles liées au capital humain - notamment le niveau d'éducation - peuvent s'avérer déterminantes (Schwartz, 1973 : 1153-1169). La part des immigrés dans la population active ainsi que leur participation au marché du travail se sont considérablement accrues durant la dernière décennie dans I'OCDE. Mais, cette augmentation cache de fortes disparités si on se réfère au critère d'instruction et d'apprentissage des migrants. L'évaluation des effets de ces flux sur 
la croissance économique demeure une question majeure dans le débat de politique économique depuis quelques années. Cet article présente un modèle généralisé, en panel dynamique, des déterminants de la croissance en incluant simultanément les facteurs traditionnels et récents ainsi que les déterminants non économiques. L'on s'interroge si la migration est bénéfique ou non aux vingt-neuf pays membres de I'OCDE durant la période 2000-2015. Faut-il alors encourager l'émigration des personnes qualifiées et compétentes et s'opposer aux moins instruits ? Les qualifications et les compétences acquises par les allochtones déterminent leur décision de migrer et affectent positivement la croissance des pays de l'OCDE. Cependant, ceux qui ont un niveau d'éducation faible présentent un impact économique insignifiant.

Enfin, les éclairages apportés par cet article montrent quel que soit le niveau d'apprentissage et de compétences acquis par les immigrés, ces derniers ont une valeur ajoutée remarquable pour les pays d'accueil. À long terme, les bénéfices générés de la migration pour une économie sont nettement plus importants que les coûts. À cause de ses effets sur la croissance, la migration reste une question qui ne doit pas être négligée sur le plan politique. Les pays d'accueil des migrants doivent-ils alors prendre en compte ces implications positives dans leurs stratégies de développement et imaginer des politiques de gestion de ces flux migratoires en accord avec leurs objectifs de politique économique ? Ou paradoxalement, doivent-ils freiner cette migration par des décisions de libre-échange et de co-développement nord-sud ? À ce niveau, une nouvelle piste de travail s'ouvre prenant en compte l'impact des quotas ou d'autres aspects sélectifs des politiques migratoires sur la croissance économique et testant avec précision la relation entre les migrations et l'âge des migrants. Plusieurs améliorations peuvent donc être attendues dans le futur, notamment grâce à une meilleure qualité des données, tant au niveau micro qu'au niveau macroéconomique. 


\section{Références bibliographiques}

Aisen Ari and Veiga Francisco Jose (2011) How does political instability affect economic growth?, Middle East and Central Asia Department, IMF Working Paper 12, 29 p.

Aizenman Joshua (1994) Monetary and real shocks, productive capacity and exchange rate regimes, Economica, 61 (244), pp. 407-434.

Akbari Ather H. and MacDonald Martha (2014) Immigration policy in Australia, Canada, New Zealand and the United States: an overview of recent trends, International Migration Review, 48 (3), pp. 577-917.

Angrist Joshua D. and Kugler Adriana D. (2003) Protective or counter-productive? Labour market institutions and the effect of immigration on the natives, Economic Journal, 113 (488), pp. 302-331.

Banque Mondiale (2018) World Development Indicators, [en ligne]. URL : https:// databank.worldbank.org/data/databases

Baltagi Badi H. Wiley (1995) Econometric analysis of panel data, Journal of Econometric Theory, 13 (5), pp. 747-754.

Barro Robert and Lee Jong-Wha (2013) A new data set of educational attainment in the world, 1950-2010, Journal of Development Economics, 104, pp. 184-198.

Barro Robert J. and Sala-I-Martin Xavier (1995) Technological diffusion, convergence, and growth, NBER Working Paper 5151, 26 p.

Beck Paul J. and Maher Michael W. (1986) A comparison of bribery and bidding in thin markets, Journal of Economics Letters, 20 (1), pp. 1-5.

Becker Gary S., Murphy Kevin N. and Tamura Robert F. (1990) Human capital, fertility, and economic growth, NBER Working Paper 3414, 44 p.

Blundell Richard and Bond Stephen (1998) Initial conditions and moment restrictions in dynamic panel data models, Journal of Econometrics, 87, pp. 115-143.

Borjas George J. (2009) The analytics of the wage effect of immigration, NBER Working Papers 14796, 54 p.

Borjas George J. (2003) The labor demand curve is downward sloping: reexamining the impact of immigration on the labor market, Quarterly Journal of Economics, $118(4)$, pp. 1335-1374

Boubtane Ekrame, Dumont Jean-Christophe and Rault Christophe (2013) Immigration and economic growth in the OECD countries 1986-2006: A panel data analysis, [online]. URL: https://halshs.archives-ouvertes.fr/ halshs-01252406/ document

Can Ensar, Ramel Nathalie und Sheldon George (2014) Effekte der Personenfreizügigkeit auf die wirtschaftliche Entwicklung der Schweiz, Forschungsstelle für Arbeitsmarkt und Industrieökonomik (FAI), [online]. URL: https://biblio.parlement.ch/e-docs/373941.pdf

Card David (1990) The impact of the Mariel boatlift on the Miami labor market, Industrial et Labor Relations Review, 43 (2), pp. 245-257. 
Chaloff Jonathan and Lemaître Georges (2009) Managing highly-skilled labour Migration: A comparative analysis of migration policies and challenges in OECD countries, OECD social, Employment and Migration Working Papers 79, Paris, OECD Publishing, $54 \mathrm{p}$.

Chojnicki Xavier et Ragot Lionel (2012) On entend dire que l'immigration coûte cher à la France. Qu'en pensent les économistes, Paris, Les Échos Éditions/ Éditions Eyrolles, 20 p.

Chui Michael, Levine Paul and Pearlman Joseph (2001) Winners and losers in a North-South model of growth, innovation and product cycles, Journal of Development Economics, 65, pp. 333-365.

Collier Paul and Gunning Jan Willem (1999) Explaining African economic performance, Journal of Economic Literature, 37 (1), pp. 64-111.

Currie David, Levine Paul, Pearlman Joseph and Chui Michael (1999) Phases of imitation and innovation in a North-South endogenous growth model, Oxford Economic Papers, 51 (1), pp. 60-88.

D'Albis Hippolyte, Boubtane Ekrame and Coulibaly Dramane (2013) Immigration et croissance économique en France entre 1994 et 2008, Working Papers, 201305, CERDI, $23 \mathrm{p}$.

Dal Bó Ernesto and Rossi Martin A. (2007) Corruption and inefficiency: Theory and evidence from electrical utilities, Journal of Public Economics, 91 (5-6), pp. 939-962.

D'Auria Francesca, Mc Morrow Kieran and Pichelmann Karl (2008) Economic impact of migration flows following the 2004 EU enlargement process: A model based analysis, Commission européenne Economic Papers 349, 34 p.

Dolado Juan, Goria Alessandra and Ichino Andrea (1994) Immigration, human capital and growth in the host country: evidence from pooled country data, Journal of Population Economics, 7 (2), pp. 193-215.

Eichengreen Barry and Dincer Nergiz (2009) Central Bank Transparency: Causes, Consequences and Updates, NBER working papers 14791, $64 \mathrm{p}$.

Eurostat (2018) Eurostatistics - Data for short-term economic analysis, 11.

European Union Agency for Fundamental Rights (2014) Council of Europe (2014), Handbook on european law relating to asylum, borders and immigration, Belgium, Publications Office of the European Union, $278 \mathrm{p}$.

FMI (2018) International Financial Statistics (IFS).

Friberg Jon H. and Eldring Line (2013) Labour migrants from central and Eastern Europe in the nordic countries patterns of migration, working conditions and recruitment practices, [en ligne]. URL: http://norden.diva-portal.org/smash/ record.jsf?pid=diva2\%3A702572\&dswid=6781

Friedberg Rachel M. (2001) The impact of mass migration on the Israeli labor market. Quarterly Journal of Economics, 116 (4), pp. 1373-1408.

Giang Ho and Shirono Kazuko (2015) The Nordic labor market and migration, IMF Working Paper, European Department, WP/15/254, 45 p.

Grossman Gene M. and Helpman Elhanan (1991) Innovation and growth in the global economy, Cambridge, Massachusetts, MIT Press, 359 p. 
Hansen Jorgen and Lofstrom Magnus (2003) Immigrant assimilation and welfare participation: Do immigrants assimilate into or out-of welfare, Journal of Human Resources, 38 (1), pp. 74-98.

Hinger Sophie (2017) Allemagne : Les villes-laboratoires, un autre accueil possible ?, [en ligne]. URL : https://www.revue-projet.com/articles/2017-juin hinger_allemagne_les-villes-laboratoires-un-autre-accueil-possible/

Honoré Renaud (2015) Entre Europe de l'est et de l'ouest, un clivage culturel sur les réfugiés, [en ligne]. URL : https://www.lesechos.fr/08/09/2015/lesechos. fr/021312991438_entre-europe-de-I-est-et-de-I-ouest--un-clivage-culturel-sur-lesrefugies.htm

Hunt Jennifer (1992) The impact of the 1962 repatriates from Algeria on the French labor market, industrial and labor relations review, 45 (3), pp. 556-572.

Ichou Mathieu (2014) Who they were there: Immigrants' educational selectivity and their children's educational attainment, European Sociological Review, 30 (6), pp. 750-765.

IOM (2018) Global Migration Indicators, [online]. URL: www.migrationdataportal. org

Ivlevs Artjoms (2006) Migration and FDI in the globalization context: The case of the small open economy, mimeo, CEDERS, University of Aix-Marseille, $15 \mathrm{p}$.

Jakob Christian (2016) Die Bleibenden, Wie Flüchtlinge Deutschland seit 20 Jahren verändern, Berlin, Ch. Links, $15 \mathrm{p}$.

Jawaid Tehseen and Raza Syed Ali (2016) Effects of workers' remittances and its volatility on economic growth in south Asia, International Migration, 54, pp. 50-68.

Jean Sébastien and Jiménez Miguel (2011) The unemployment impact of immigration in OECD countries, European Journal of Political Economy, 27 (2), pp. 241-256.

Khan Mohsen S. and Senhadji Abdelhak S. (2000) Threshold effects in the relationship between inflation and growth, IMF working papers 110, $32 \mathrm{p}$.

Kharlamova Ganna, Sitnitskiy Maksym (2016) Growing regional scientific migration and mobility: the European Union and the eastern partnership, [online]. URL: http://cse.uaic.ro/eurint/proceedings/index_htm_files/EURINT\%20 2016_KHA.pdf

Kingsley Davis (1974) The Migration of human populations, Scientific American, 231 (3), pp. 92-107.

Lambsdorff Johann Graf (2003) How corruption affects productivity, Kyklos, 56 (4), pp. 457-474.

Levine Paul, Lotti Emanuela, Pearlman Joseph and Pierse Richard Gerard (2010) Growth and welfare effects of world migration, Scottish Journal Of Political Economy, 57 (5), pp. 615-643.

Lisenkova Katerina, Mérette Marcel and Sanchez-Martinez Miguel (2014) The long-term economic impact of reducing migration in the UK, National Institute Economic Review, 229 (22), pp. 22-30. 
Longhi Simonetta, Nijkamp Peter and Poot Jacques (2006) The impact of immigration on the employment of natives in regional labour markets: a meta-analysis, ISER Working Paper 10, 26 p.

Lubotsky Darren (2007) Chutes or ladders? A longitudinal analysis of immigrant earnings, Journal of Political Economy, 115 (5), pp. 820-867.

Lucas Robert E. (1988) On the mechanics of economic development, Journal of Monetary Economics, 22, pp. 3-42.

Mankiw Gregory N., Romer David and Weil David N. (1992) A Contribution to the empirics of economic growth, The Quarterly Journal of Economics, 107 (2), pp. 407-437.

Monnet Jérôme (2006) Le Mexique face aux États-Unis : 1906-2006, un siècle de révolutions, in 17ème Festival International de Géographie, Les géographes revisitent les Amériques, Saint-Dié des Vosges, [en ligne]. URL : http://archivesfig-st-die.cndp.fr/actes/actes_2006/monnet/mexique-etats-unis.pdf

Monso Olivier et Gleizes François (2009) Langue, diplômes : des enjeux pour I'accès des immigrés au marché du travail, Division Emploi INSEE, Publications Grand Public, 1262, 4 p.

Mouhoud El Mouhoub (2017) L'immigration en France, mythes et réalités, Paris, Fayard, 200 p.

Mubarik Yasir Ali (2005) Inflation and growth: An estimate of the threshold level of inflation in Pakistan, State Bank of Pakistan Research Bulletin 1, pp. 35-44

Pan Ké Shon Jean-Louis and Verdugo Gregory (2015) Forty years of immigrant segregation in France, 1968-2007. How different is the new immigration?, Urban Studies Journal, 52 (5), pp. 823-840.

Picot Garnett, Hou Feng et Qiu Theresa (2014) Le modèle de sélection selon le capital humain et les résultats économiques à long terme des immigrants, Statistique Canada, Direction des études analytiques, document de recherche $11 \mathrm{~F} 0019 \mathrm{~N}, 361$.

OCDE (2018) Economic Outlook, 103.

OCDE (2016) Economic Outlook, 100.

OCDE (2016) Immigrant and foreign population, OCDE Factbook 2015-2016: Economic, Environmental and Social Statistics, [online]. URL: https://read.oecd-ilibrary.org/economics/oecd-factbook-2015-2016/immigrant-and-foreign-population_factbook-2015-5-en\#page1

OCDE (2015) Economic Outlook, 98.

OCDE (2015) Perspectives des migrations internationales, [en ligne]. URL : https:// books.google.fr/books/about/Perspectives_des_migrations_internationa.html ?id=DWyXCgAAQBAJ\&printsec=frontcover\&source=kp_read_button\&redir_ esc $=\mathrm{y} \# \mathrm{v}=$ onepage $\& \mathrm{q} \& \mathrm{f}=\mathrm{false}$

OCDE (2013) Perspectives des migrations internationales, [en ligne]. URL : https://books.google.fr/books/about/Perspectives_des_migrations_internationa. html?id=m8c7A0rq7PwC\&printsec=frontcover\&source=kp_read_button\&redir_ esc $=\mathrm{y} \# \mathrm{v}=$ onepage $\& \mathrm{q} \& \mathrm{f}=\mathrm{false}$ 
OCDE (2002) Prendre la mesure du travail temporaire, in OCDE Éd., Perspectives de l'emploi de l'OCDE 2002, [en ligne]. URL : http://www.oecd.org/fr/els/ emp/19147433.pdf

Ortega Francesc and Peri Giovanni (2009) The causes and effects of international migrations: Evidence from OECD countries 1980-2005, NBER working papers $14833,42 \mathrm{p}$.

Ortega Javier and Verdugo Gregory (2011) Immigration and the occupational choice of natives: a factor proportions approach, Banque de France Working papers $335,45 \mathrm{p}$.

Ottaviano Gianmarco and Peri Giovanni (2008) Immigration and national wages: clarifying the theory and the empirics, NBER Working Papers 14188, 68 p.

Schuss Eric (2016) Between life cycle model, labor market integration and discrimination: an econometric analysis of the determinants of return migration, SOEP papers on Multidisciplinary Panel Data Research 881, 30 p.

Schwartz Aba (1973) Interpreting the effect of distance on migration, Journal of Political Economy, 81, pp. 1153-1169.

Stalder Peter (2010) Free migration between the EU and Switzerland: impacts on the Swiss economy and implications for monetary policy, Swiss Journal of Economics and Statistics (SJES), Swiss Society of Economics and Statistics (SSES), 146 (IV), pp. 821-874.

Steiner Ilka, Watkins Aronne et D’Amato Gianni (2013) Démographie, ressources et la signification de la migration, Confédération suisse, Commission fédérale pour les questions de migration CFM, [online]. URL: https://www.ekm.admin.ch/ $\mathrm{dam} / \mathrm{data} / \mathrm{ekm} /$ dokumentation/materialien/mat_demographie_f.pdf

Storesletten Kjetil (2003) Fiscal implications of immigration. À net present value approach, Scandinavian Journal of Economics, 105 (3), pp. 487-506.

Suter Stephan (2006) Bildung oder Begabung? Ökonometrische Untersuchungen zu den Ursachen, Hamburg, Dr. Kovac, 162 p.

Talvi Ernesto and Vegh Carlos (2005) Tax base variability and procyclical fiscal policy in developing countries, Journal of Development Economics, 78 (1), pp. 156-190.

The World Bank Group (2018) Worldwide Governance Indicators Project [online]. URL: https://info.worldbank.org/governance/wgi/index.aspx\#home

Vandenbussche Jérôme, Aghion Philippe and Meghir Costas (2006) Growth, distance to frontier and composition of human capital, Journal of Economic Growth, 11 (2), pp. 97-127.

White Halbert (1980) A heteroskedasticity-consistent covariance matrix estimator and a direct test for heteroskedasticity, Econometrica, 48 (4), pp. 817-838. 


\section{Annexes}

\section{Synthèse technique}

\section{Présentation des scénarii}

Pour répondre à notre problématique, nous effectuons quatre premiers essais différents dont les variables endogènes sont respectivement la part des migrants dans la population totale ( $P_{-}$mgr), la part de migrants ayant un niveau d'éducation élevé (E_haut), la part de migrants ayant un niveau d'éducation moyen ( $E_{-}$moyen) et la part de migrants ayant un niveau d'éducation faible ( $E_{-}$ bas). Au niveau de chaque scénario, nous testons, en plus des variables macroéconomiques classiques, l'impact de chacune desdites variables de migration sur la croissance économique des pays de I'OCDE.

Dans un second temps, nous réalisons un cinquième essai qui constitue un scénario démonstratif et témoin. Ce scénario englobe simultanément les trois variables représentatives du capital humain par niveau d'éducation (E_haut, $E_{-}$ moyen et E_bas). L'objet de ce choix est de déterminer laquelle de ces variables de migration qui impacte davantage le PIB per capita.

\section{Échantillon}

Allemagne, Australie, Autriche, Belgique, Canada, Danemark, Espagne, Estonie, États-Unis, Finlande, France, Grèce, Hongrie, Irlande, Islande, Italie, Luxembourg, Norvège, Nouvelle-Zélande, Pays-Bas, Pologne, Portugal, République slovaque, République tchèque, Royaume-Uni, Slovénie, Suède, Suisse, Turquie.

\section{Équation de la croissance}

L'équation de la croissance dépend du capital humain, représenté par la part des migrants en fonction de leur niveau d'éducation, et un ensemble de variables de contrôle afin de tenir compte des effets potentiels des autres déterminants de la croissance. On se réfère dans le choix desdites variables aux travaux de Vandebussche et al. (2006: 97-127), Grossman et Helpman (1991), Currie et al. (1999: 60-88), Chui et al. (2001 : 333-365), Levine et al. (2010:615-643) et Barro et Lee (2013: 184-198). Cette équation se présente comme suit :

$$
\begin{aligned}
& \mathrm{PIB}_{i, \mathrm{t}}=\alpha_{1} \mathrm{PIB}_{\mathrm{i}, \mathrm{t}-1}+\alpha_{2} \mathrm{ACC}_{\mathrm{i}, \mathrm{t}}+\alpha_{3} K_{i, t}+\alpha_{4} \mathrm{INFL}_{\mathrm{i}, \mathrm{t}}+\alpha_{5} \mathrm{OUV}_{\mathrm{i}, \mathrm{t}}+\alpha_{6} \mathrm{I}_{\mathrm{i}, \mathrm{t}}+\alpha_{7} \mathrm{IDE}_{\mathrm{i}, \mathrm{t}}+\alpha_{8} \text { FONDS }_{\mathrm{i}, \mathrm{t}} \\
& +\alpha_{9} \mathrm{G}_{\mathrm{i}, \mathrm{t}}+\alpha_{10} \mathrm{CC}_{\mathrm{i}, \mathrm{t}}+\varepsilon_{\mathrm{i}, \mathrm{t}}
\end{aligned}
$$

\section{Outil économétrique}

L'exercice empirique se base sur la méthode des moments généralisés en système dans le cadre des panels dynamiques (Blundel et Bond, 1998 : 115-143). Les observations sont annuelles et décrivent la période 2000-2015. 


\section{Détails des variables utilisées}

Tableau 3 : Description des données et des sources

\begin{tabular}{|c|c|}
\hline Variables & Définitions \\
\hline \multicolumn{2}{|l|}{ Variable dépendante } \\
\hline $\mathrm{PIBi}, \mathrm{t}$ : & Taux de croissance du PIB réel per capita \\
\hline \multicolumn{2}{|l|}{ Variables indépendantes } \\
\hline $\begin{array}{l}\text { ACCi,t : Taux d'accroissement } \\
\text { naturel de la population }\end{array}$ & $\begin{array}{l}\text { Différence entre le nombre de naissances et le nombre de décès } \\
\text { Le signe attendu de cette variable est positif }\end{array}$ \\
\hline $\begin{array}{l}\text { KHi,t : Capital humain des } \\
\text { migrants }\end{array}$ & $\begin{array}{l}\text { Part de la masse migrante dans la population totale en fonction du } \\
\text { niveau d'éducation (Suter, } 2008 \text {; Can et al., 2014) } \\
\text { P-mrg : tous ; E_haut ; E_moyen ; E-bas } \\
\text { Le signe attendu pour chaque variable en jeu est à déterminer }\end{array}$ \\
\hline INFLi,t : Taux d'inflation & $\begin{array}{l}\text { L'inflation défavorise les investissements à long terme et réduit la } \\
\text { croissance (Khan et Senhadji, } 2000 \text {; Mubarik, } 2005: 35-44 \text { ) } \\
\text { Le signe attendu est } \underline{\text { négatif }}\end{array}$ \\
\hline $\begin{array}{l}\text { OUVi,t : Degré d'ouverture } \\
\text { au commerce et aux flux de } \\
\text { capitaux internationaux }\end{array}$ & $\begin{array}{l}\text { Ratio de la somme des exportations et des importations au PIB } \\
\text { Le signe attendu est positif }\end{array}$ \\
\hline $\begin{array}{l}\text { li,t : Investissements publics } \\
\text { et privés }\end{array}$ & L'investissement a fort effet positif (Aizenman, 1994 : 407-434) \\
\hline $\begin{array}{l}\text { IDEi,t : Investissements } \\
\text { directs étrangers }\end{array}$ & $\begin{array}{l}\text { L'effet des IDE est positif sur la croissance grâce aux transferts de } \\
\text { technologie induits }\end{array}$ \\
\hline $\begin{array}{l}\text { Gi,t : Dépenses publiques } \\
\text { réelles dans le PIB }\end{array}$ & $\begin{array}{l}\text { Sans influence directe sur la productivité, elles peuvent fausser les } \\
\text { décisions du secteur privé (Barro et Sala-I-Martin, 1995) } \\
\text { Le coefficient de cette variable est négatif }\end{array}$ \\
\hline $\begin{array}{l}\text { FONDSi,t : Transferts de } \\
\text { fonds des migrants }\end{array}$ & $\begin{array}{l}\text { L'impact de cette variable sur le pays de destination reste } \\
\text { controversé }\end{array}$ \\
\hline $\begin{array}{l}\text { CCi,t : Contrôle de la } \\
\text { corruption }\end{array}$ & $\begin{array}{l}\text { Il mesure I'usage des prérogatives du pouvoir à des fins } \\
\text { personnelles, en particulier l'enrichissement des individus disposant } \\
\text { d'une position de pouvoir. Le contrôle de la corruption permet } \\
\text { d'améliorer l'efficacité économique (Beck et Maher, 1986: } 1-5 \text {; } \\
\text { Lambsdorff, } 2003: 457-474 \text {; Dal Bó et Rossi, } 2007: 939-962 \text { ). Le } \\
\text { signe attendu est positif. }\end{array}$ \\
\hline
\end{tabular}

Sources : IOM, 2018 ; Banque mondiale, 2018 ; OCDE, 2018 ; FMI, 2018 ; Eurostat, 2018 ;

The World Bank Group, 2018.

\section{Tests économétriques}

Les tests ci-dessous garantissent la fiabilité du modèle choisi et permettent d'accomplir les corrections nécessaires.

\section{Autocorrélation des variables}

L'autocorrélation (ou l'autocovariance) d'une série fait référence au fait que dans une série temporelle ou spatiale, la mesure d'un phénomène à un instant $t$ peut être corrélée aux mesures précédentes $(\mathrm{t}-1, \mathrm{t}-2, \mathrm{t}-3$, etc.) ou aux mesures suivantes $(t+1, t+2, t+3$, etc.). La présence de ce problème dans un modèle produit des résultats erronés et falsifie les interprétations économiques. La matrice de corrélation, présentée en annexe (tableau 4), permet d'examiner l'existence ou non d'une autocorrélation entre les variables. On a effectué des tests d'autocorrélation des dix variables en jeu et on a déduit que la part des migrants dans la population totale (P_mgr) est corrélée avec la variable contrôle de corruption (CC) et avec les trois proportions de migrants en fonction de leur 
niveau d'enseignement acquis (E_haut, E_moyen et $E_{-}$bas). Ces derniers ne sont pas corrélés entre eux, ce qui permet de conclure quant à l'inexistence du problème de multi-colinéarité.

Test de stationnarité des variables

L'étude de la stationnarité des variables permet d'avoir une idée sur les caractéristiques des séries étudiées. Elle permet également d'éviter les régressions fallacieuses. Pour s'assurer de la robustesse des résultats de stationnarité des différentes variables en jeu, on a opté pour le test (Pesaran, 2003) qu'on a effectué en présence et en absence de tendance. Les variables PIB, INFL, IDE, OUV, G, I et FONDS sont stationnaires en niveau alors que CC, ACC et P_mgr sont intégrées d'ordre un. E_haut, E_moyen et E_bas sont intégrées d'ordre deux.

Endogénéité des variables : variables instrumentales

Pour résoudre le problème d'endogénéité qui peut apparaître dans le modèle, et pour déterminer la meilleure spécification du modèle, on utilise une ou plusieurs variables instrumentales corrélées avec la variable endogène et non corrélée avec les résidus. Le test de sur-identification de Sargan nous a permis d'accepter la validité de toutes les variables de contrôle comme instruments. On se réfère aux variables instrumentales (Baltagi, 1995 : 747-754).

Test d'hétéroscédasticité des erreurs

Pour assurer la robustesse des résultats lors de l'estimation, et que le terme d'erreur de notre modèle est homoscédastique (variance constante des résidus) et que les coefficients sont efficaces, on effectue le test d'Eicker et White (1980: 817-838) pour corriger I'hétéroscédasticité des erreurs.

\section{Tableaux}

Tableau 4 : Matrices des corrélations

\begin{tabular}{llllll}
\hline & P_mgr & E_haut & E_moy & E_bas & CC \\
\hline P_mgr & 1.0000 & & & & \\
E_haut & 0.7557 & 1.0000 & & & \\
E_moy & 0.8832 & & 1.0000 & & \\
E_bas & 0.7651 & & & 1.0000 & \\
CC & 0.5027 & & & & 1.0000 \\
\hline
\end{tabular}

Légende : P-mrg : tous niveaux d'instruction; E_haut : niveau d'instruction élevé ;

E_moyen : niveau d'instruction moyen; E-bas : niveau d'instruction bas.

Sources : OCDE, 2018 ; Eurostat, 2018 ; The World Bank Group, 2018. 
Tableau 5 : Résultats des estimations du modèle de croissance

\begin{tabular}{|c|c|c|c|c|c|}
\hline $\begin{array}{l}\text { Variable dépendante : tauX } \\
\text { de croissance du PIB per } \\
\text { capita réel }\end{array}$ & Scénario (1) & Scénario (2) & Scénario (3) & Scénario (4) & Scénario (5) \\
\hline yt-1 & $\begin{array}{c}0,106 \\
(0,393)\end{array}$ & $\begin{array}{l}0,463^{*} \\
(0,028)\end{array}$ & $\begin{array}{c}0,105 \\
(0,400)\end{array}$ & $\begin{array}{c}0,096 \\
(0,450)\end{array}$ & $\begin{array}{l}-0,074^{*} \\
(-0,056)\end{array}$ \\
\hline Accroissement naturel & $\begin{array}{c}8.90378^{*} \\
(0,022)\end{array}$ & $\begin{array}{c}7.09454^{*} \\
(0,051)\end{array}$ & $\begin{array}{c}9.13156^{*} \\
(0,030)\end{array}$ & $\begin{array}{l}9167^{*} \\
(0,042)\end{array}$ & $\begin{array}{c}9088^{* * *} \\
(0,000)\end{array}$ \\
\hline \multicolumn{6}{|l|}{ Capital humain étranger: } \\
\hline 1. P_img & $\begin{array}{c}1626 * * * \\
(0,000)\end{array}$ & & & & \\
\hline 2. E_haut & & $\begin{array}{c}6.07341 * * * \\
(0,000)\end{array}$ & & & $\begin{array}{c}2188^{* * * *} \\
(0,000)\end{array}$ \\
\hline 3. E_moyen & & & $\begin{array}{c}0.57865^{* *} \\
(0,013)\end{array}$ & & $\begin{array}{c}2632 * * * \\
(0,000)\end{array}$ \\
\hline 4. E_bas & & & & $\begin{array}{c}0.82951 * * * \\
(0,000)\end{array}$ & $\begin{array}{c}1232 \\
(0,301)\end{array}$ \\
\hline Investissement & $\begin{array}{c}1.73029 * * * \\
(0,000)\end{array}$ & $\begin{array}{c}0.62568 \\
(0,113)\end{array}$ & $\begin{array}{c}2.64830 * * * \\
(0,000)\end{array}$ & $\begin{array}{c}-2 \cdot 13897^{* * *} \\
(0,000)\end{array}$ & $\begin{array}{c}-1.81122^{* * *} \\
(0,000)\end{array}$ \\
\hline $\begin{array}{l}\text { Investissement direct } \\
\text { étranger }\end{array}$ & $\begin{array}{c}0.22088^{* * *} \\
(0,000)\end{array}$ & $\begin{array}{c}0.20959 * * \\
(0,003)\end{array}$ & $\begin{array}{c}0.12096^{* *} \\
(0,004)\end{array}$ & $\begin{array}{c}-0.16805 \\
(0,135)\end{array}$ & $\begin{array}{c}0.06046 \\
(0,190)\end{array}$ \\
\hline Dépenses publiques & $\begin{array}{c}0.22088^{* * *} \\
(0,000)\end{array}$ & $\begin{array}{c}0.20543^{* * *} \\
(0,000)\end{array}$ & $\begin{array}{c}-0.46161 \\
(0,383)\end{array}$ & $\begin{array}{c}0.08853^{* * *} \\
(0,000)\end{array}$ & $\begin{array}{c}0.89554^{* * *} \\
(0,000)\end{array}$ \\
\hline Ouverture commerciale & $\begin{array}{l}8,68 * * * \\
(0,000)\end{array}$ & $\begin{array}{l}8,66^{* *} \\
(0,004)\end{array}$ & $\begin{array}{l}5,77 * * \\
(0,001)\end{array}$ & $\begin{array}{l}2,46^{*} \\
(0,094)\end{array}$ & $\begin{array}{l}4751 * * \\
(0,007)\end{array}$ \\
\hline Inflation & $\begin{array}{l}1,94 * * \\
(0,004)\end{array}$ & $\begin{array}{l}-3,97 \\
(0,742)\end{array}$ & $\begin{array}{l}-1,50^{*} \\
(0,021)\end{array}$ & $\begin{array}{c}1,14 \\
(0.1002)\end{array}$ & $\begin{array}{l}2118^{* *} \\
(0,004)\end{array}$ \\
\hline $\begin{array}{l}\text { Transferts de fonds des } \\
\text { immigrés }\end{array}$ & $\begin{array}{c}0.30409 * * * \\
(0,000)\end{array}$ & $\begin{array}{c}0.58964^{* * *} \\
(0,000)\end{array}$ & $\begin{array}{c}-0.29749 * * * \\
(0,000)\end{array}$ & $\begin{array}{c}-0.23644^{* * *} \\
(0,000)\end{array}$ & $\begin{array}{c}0.32728^{* * *} \\
(0,000)\end{array}$ \\
\hline Contrôle de corruption & & $\begin{array}{c}0.22194^{* *} \\
(0,002)\end{array}$ & $\begin{array}{c}0.57691 * * * \\
(0,000)\end{array}$ & $\begin{array}{c}0.59763^{* * *} \\
(0,000)\end{array}$ & $\begin{array}{c}-0.3107^{* * *} \\
(0,000)\end{array}$ \\
\hline Constante & $\begin{array}{c}8.31199 * * * \\
(0,000)\end{array}$ & & $\begin{array}{c}10.82721 * * * \\
(0,000)\end{array}$ & $\begin{array}{c}10.02109 * * * \\
(0,000)\end{array}$ & $\begin{array}{c}9.93525^{* * *} \\
(0,000)\end{array}$ \\
\hline Nombre d'observations & 407 & 392 & 392 & 392 & 392 \\
\hline Wald chi2 & 597,14 & 694,63 & 725,23 & 735,19 & 740,63 \\
\hline Prob $>$ chi2 & 0.0000 & 0.0000 & 0.0000 & 0.0000 & 0.0000 \\
\hline
\end{tabular}

Légende : Les valeurs entre parenthèses sont des p-values. ${ }^{*},{ }^{* *},{ }^{* * *}$ indiquent que les coefficients sont respectivement significatifs aux seuils $10 \%, 5 \%$ et $1 \%$. Sources : OCDE, 2018; The World Bank Group, 2018 ; Eurostat, 2018. 


\section{Leila Ben Ltaief}

\section{Impact de la migration sur la croissance économique dans les pays de I'OCDE}

Cet article étudie le dynamisme de la migration internationale et ses conséquences sous-jacentes sur le marché du travail et le chômage. Empiriquement, on présente une évaluation de l'impact de ce phénomène sur la croissance économique de vingt-neuf pays de l'OCDE durant la période 2000-2015. Ce travail repose sur un ensemble de données qui permet de distinguer les migrants selon leur niveau d'éducation. Cette structure constitue une caractéristique essentielle qui détermine en grande partie les principaux effets sur la croissance économique. L'outil économétrique est basé sur la méthode des moments généralisés en panel dynamique afin de pouvoir traiter l'endogénéité potentielle des variables de migration. Dans ce cadre, nous identifions un impact positif du capital humain étranger sur la croissance économique des pays d'accueil, à l'exception de celui qui représente les allochtones faiblement instruits.

\section{Impact of Immigration on Economic Growth in the OECD Countries}

This article examines the international dynamics of immigration and its underlying implications on labour market and unemployment. Empirically, we present a reappraisal of the migration impact on economic growth for twenty-nine OECD countries during the period 2000-2015. It is based on a dataset that enables to distinguish migration by education level. This structure is an essential feature that largely determines the main effects on economic growth. The econometric tool is based on the Generalized Method of Moments for dynamic panel, in order to deal with the potential endogeneity of the migration variables. In this framework, we identify a positive impact of the foreign human capital on economic growth of the receiving countries, with the exception of one who represents immigrants weakly educated.

\section{Impacto de la inmigración sobre el crecimiento económico en los países de la OCDE}

Este artículo examina la dinámica internacional de la inmigración y sus implicaciones subyacentes en el mercado laboral y el desempleo. Empíricamente, presentamos una reevaluación del impacto de la migración en el crecimiento económico de veintinueve países de la OCDE durante el período 2000-2015. Se basa en un conjunto de datos que permite distinguir la migración por nivel de educación. Esta estructura es una característica esencial que determina en gran medida los principales efectos sobre el crecimiento económico. La herramienta econométrica se basa en el método generalizado de estimaciones de momento para panel dinámico, con el fin de abordar la endogeneidad potencial de las variables de migración. En este marco, identificamos un impacto positivo del capital humano extranjero en el crecimiento económico de los países receptores, con la excepción de uno que representa a los inmigrantes con poca educación. 